Sammlung Metzler Band 307 
Achim Stein

\section{Einführung in die}

französische

Sprachwissenschaft

Verlag J.B. Metzler Stuttgart - Weimar 


\section{Angaben zum Autor}

Achim Stein, geb. 1962; Promotion 1993. Seit 1989 wissenschaftlicher Assistent an der Universität Stuttgart.

Die Deutsche Bibliothek - CIP-Einheitsausnahme

\section{Achim Stein:}

Einführung in die französische Sprachwissenschaft

/ Achim Stein.

- Stuttgart ; Weimar : Metzler, 1998

(Sammlung Metzler ; Bd. 307)

ISBN 978-3-476-10307-9

NE: GT

\section{SM 307}

ISBN 978-3-476-10307-9

ISBN 978-3-476-04024-4 (eBook)

DOI 10.1007/978-3-476-04024-4

ISSN 05583667

Dieses Werk einschließlich aller seiner Teile ist urheberrechtlich geschützt. Jede Verwertung außerhalb der engen Grenzen des Urheberrechtsgesetzes ist ohne Zustimmung des Verlages unzulässig und strafbar. Das gilt insbesondere für Vervielfältigungen, Übersetzungen, Mikroverfilmungen und die Einspeicherung und Verarbeitung in elektronischen Systemen.

(C) 1998 Springer-Verlag GmbH Deutschland

Ursprünglich erschienen bei J.B. Metzlersche Verlagsbuchhandlung und Carl Ernst Poeschel Verlag GmbH in Stuttgart 1998 


\section{Vorwort}

Dieses Buch ist aus den Einführungskursen in die französische Sprachwissenschaft an der Universität Stuttgart hervorgegangen. Es ist so aufgebaut, daß es solchen Kursen als Grundlage dienen und im Rahmen eines Semesterprogramms durchgearbeitet werden kann. Da es außer einigen gängigen Begriffen der Grammatik keine besonderen Vorkenntnisse voraussetzt, ist es auch zum Selbststudium geeignet.

Den Kern des Buchs bilden knapp 700 Termini aus den verschiedenen linguistischen Bereichen. Aus Gründen der Übersichtlichkeit sind ihre französischen Übersetzungen nicht im Text genannt, sondern in den zweisprachigen Sachregistern (deutsch-französisch und französisch-deutsch). Sie ermöglichen das schnelle Nachschlagen oder die Kontrolle des Fachwortschatzes vor Prüfungen.

Ich habe mich bemüht, die Kapitel und die Themenbereiche innerhalb der Kapitel sinnvoll anzuordnen, und empfehle, das Buch in dieser Reihenfolge durchzuarbeiten. Kapitel können aber auch isoliert bearbeitet werden, wenn unbekannte Termini bei Bedarf über das Sachregister nachgeschlagen werden. Die systematische Nennung weiterführender Literatur am Ende jedes Kapitels erleichtert die Vertiefung der Lektüre. Aus Platzgründen konnten nicht alle Bereiche Eingang finden, in denen die Linguistik sich mit anderen Disziplinen überschneidet, wie die Psycholinguistik oder die Soziolinguistik. Dafür sind in Kapitel 10 einige Bereiche kurz angesprochen, die zum Teil erst in letzter Zeit für die Linguistik relevant geworden sind, wie die Korpuslinguistik und Anwendungen, die auf der Grenze zwischen Linguistik und maschineller Sprachverarbeitung angesiedelt sind. Dem Risiko der Kurzlebigkeit dieser Inhalte steht entgegen, daß zumindest ihre Existenz und die wichtigsten Grundbegriffe den Studierenden in einer Zeit der zunehmenden Öfnung der Geisteswissenschaften anderen Disziplinen gegenüber bekannt sein sollte.

Für Unterstützung und Hinweise bei der Fertigstellung dieses Buchs danke ich Peter Blumenthal, Gabi Böhme, Brigitte Martin, Jutta Rösner, Patrick Saint-Dizier, Leo Wanner, Judith Yacar und Michael Zock, auBerdem Dagmar Mann und Claudia Fischer für die Durchsicht des Manuskripts, Michael Jessen und Marcus Fach vom Institut für Experimentelle Phonetik für die Herstellung des Sonagramms und Matthias Pfisterer für die Hilfe bei der Herstellung der Druckdaten. 


\section{Inhaltsverzeichnis}

1. Sprache und Sprachwissenschaft . . . . . . . . . 1

1.1 Sprechen, Sprachen und Sprachfamilien . . . . . . . 1

1.2 Die Entwicklung der Sprachwissenschaft . . . . . . 2

1.3 Prinzipien der strukturalistischen Linguistik . . . . . 7

1.4 Literatur . . . . . . . . . . . . . . . . . . 12

2. Phonetik und Phonologie . . . . . . . . . . . . 13

2.1 Grundlagen . . . . . . . . . . . . . 13

2.2 Phonetik . . . . . . . . . . . . . . 14

2.2.1 Laute und ihre Merkmale . . . . . . . . . 14

2.2 .2 Prosodie . . . . . . . . . . . . 16

2.3 Phonologie . . . . . . . . . . . . . . . 17

2.4 Exkurs: Lautung und Schreibung $\ldots \ldots \ldots$

2.5 Literatur . . . . . . . . . . . 22

3. Morphologie . . . . . . . . . . . . . . 23

3.1 Grundbegriffe . . . . . . . . . . . . . . 23

3.2 Typen von Morphemen . . . . . . . . . . . . 25

3.3 Sprachtypen . . . . . . . . . . . . . . . . . 27

3.4 Flexion . . . . . . . . . . . . . . . . . . . . . . . 29

3.5 Wortbildung. . . . . . . . . . . . 30

3.5 .1 Grundbegriffe . . . . . . . . . . . . . 30

3.5 .2 Derivation . . . . . . . . . . . . 31

3.5 .3 Komposition . . . . . . . . . . . . 32

3.5.4 Weitere Wortbildungstypen . . . . . . . . 35

3.6 Literatur . . . . . . . . . . . . . . 36

4. Syntax . . . . . . . . . . . . . . . . . 37

4.1 Vom Satz zur Syntax . . . . . . . . . . . . . 37

4.2 Syntaktische Strukturen . . . . . . . . . . . . . 40

4.2.1 Dependenz und Valenz . . . . . . . . . . . 40

4.2.2 Konstituenz . . . . . . . . . . . . . . . . . 43

4.2.3 Syntaktische Modelle . . . . . . . . . . . . 48

4.3 Aspekte der französischen Syntax . . . . . . . . . . 50

4.3.1 Grammatische Funktionen . . . . . . . . . . 50

4.3.2 Besondere Satzgliedstellungen . . . . . . . . 52

4.4 Literatur . . . . . . . . . . . . . . . 54 
5. Semantik .................... 55

5.1 Lexikalische Semantik . . . . . . . . . 55

5.1.1 Semantische Merkmale . . . . . . . . 55

5.1.2 Das triadische Zeichenmodell . . . . . . 58

5.1.3 Prototypensemantik .......... 61

5.2 Lexikalische Relationen . . . . . . . . . . . . . 62

5.2.1 Paradigmatik ........... . . 62

5.2.2 Syntagmatik ............ 65

5.2.3 Wissensrepräsentation ......... 70

5.3 Pragmatik ............... 72

5.3.1 Sprechakte ............ 72

5.3.2 Assertion und Präsupposition . . . . . . . 74

5.4 Literatur . . . . . . . . . . . . . 76

6. Textlinguistik . . . . . . . . . . . . 78

6.1 Sinnzusammenhänge . . . . . . . . . . 78

6.2 Information im Text . . . . . . . . . . . . 81

6.3 Klassifizierung von Texten . . . . . . . . 83

6.4 Literatur . . . . . . . . . . . . . 85

7. Sprachgeschichte ............. 86

7.1 Einführung ............. 86

7.2 Die Herausbildung des Französischen . . . . . . . 87

7.3 Altfranzösisch . . . . . . . . . . . . . . 91

7.3.1 Lautentwicklung . . . . . . . . . . . 91

7.3.2 Morphologie und Syntax . . . . . . . . . 93

7.4 Mittelfranzösisch . . . . . . . . . . . . 95

7.4.1 Probleme der Periodisierung . . . . . . . . 95

7.4.2 Lautentwicklung . . . . . . . . . . . . 98

7.4.3 Schreibung und Schrift . . . . . . . . . . . 98

7.4.4 Morphologie und Syntax . . . . . . . . . 101

7.4.5 Wortschatzentwicklungen . . . . . . . . 102

7.5 Frühneufranzösisch: Das 16. Jahrhundert . . . . . 102

7.6 Neufranzösisch . . . . . . . . . . . . 105

7.6.1 Das 17. Jahrhundert . . . . . . . . . 105

7.6.2 Purismus ............. . 106

7.6.3 Vom 18. Jahrhundert bis heute . . . . . . . . 107

7.7 Literatur . . . . . . . . . . . . . . . . 109

8. Wörter und Wörterbücher . . . . . . . . . . . 111

8.1 Lexikologie . . . . . . . . . . . . . 111 
8.1.1 Historische Aspekte des Wortschatzes . . . . 111

8.1.2 Synchrone Aspekte des Wortschatzes . . . . 117

8.2 Lexikographie . . . . . . . . . . . . . 123

8.2.1 Grundbegriffe . . . . . . . . . . . 123

8.2.2 Die Entwicklung der Lexikographie . . . . . 123

8.2.3 Typen von Wörterbüchern . . . . . . . . . . 127

8.3 Literatur . . . . . . . . . . . . . 131

9. Varietäten des Französischen . . . . . . . . . . . . . 133

9.1 Norm, Varietät und Sprachpolitik . . . . . . . . . . 133

9.2 Diatopische Varietäten in Frankreich . . . . . . . . . 136

9.2.1 Die Herausbildung der dialektalen Gliederung 136

9.2.2 Die Durchsetzung des Standards . . . . . . . 137

9.2.3 Die räumliche Gliederung heute . . . . . . . 139

9.2.4 Exkurs ins Elsässische . . . . . . . . . . . . 142

9.3 Das Französische außerhalb Frankreichs . . . . . . . 143

9.3.1 Definition und Entstehung . . . . . . . . . . 143

9.3.2 Frankophonie als politischer Begriff . . . . . 145

9.3.3 Sprachkontakt und Kontaktsprachen . . . . . 146

9.3.4 Exkurs ins Québécois . . . . . . . . . . . . 148

9.4 Sprache und Gesellschaft . . . . . . . . . . . . . 152

9.4.1 Grundzüge der Soziolinguistik . . . . . . . . 152

9.4 .2 Der Faktor Alter . . . . . . . . . . . . . . . 154

9.4.3 Der Faktor Geschlecht . . . . . . . . . . . 158

9.4.4 Fach- und Sondersprachen . . . . . . . . . 159

9.4.5 Argot .............. 162

9.5 Qualitative Register . . . . . . . . . . . . . 163

9.5.1 Begriff und Geschichte . . . . . . . . . 163

9.5.2 Das français cultivé . . . . . . . . . . . . . . . 165

9.5.3 Das français familier . . . . . . . . . . . . 167

9.5.4 Das français populaire . . . . . . . . . . . . . 168

9.5.5 Das français vulgaire . . . . . . . . . . . . 170

9.6 Literatur . . . . . . . . . . . . . . 170

10. Neuere Forschungen . . . . . . . . . . . . . . 172

10.1 Anwendungen aus der Sprachverarbeitung . . . . . . 172

10.1.1 Spracherkennung . . . . . . . . . . 172

10.1.2 Morphologische Analyse . . . . . . . . 174

10.1.3 Syntaktische Analyse . . . . . . . . . . 175

10.1.4 Semantik . . . . . . . . . . . . . 175

10.1.5 Textgenerierung . . . . . . . . . . 177 
10.2 Textkorpora . . . . . . . . . . . . . 177

10.2.1 Die Entwicklung der Korpuslinguistik . . . . 177

10.2.2 Maschinenlesbare Korpora . . . . . . . . . . 179

10.2.3 Der Trésor de la langue française . . . . . . 180

11.Literatur . . . . . . . . . . . . . . . 182

Abbildungsverzeichnis . . . . . . . . . . . 196

Personenregister . . . . . . . . . . . . . 197

Sachregister Deutsch-Französisch . . . . . . . . . 199

Index des termes français-allemand . . . . . . . . 211 